\title{
Article
}

\section{Graphene Oxide/Reduced Graphene Oxide Enhanced Noniridescent Structural Colors Based on Silica Photonic Spray Paints with Improved Mechanical Robustness}

\author{
Jiali Yu (), Cheng-Hao Lee $(1)$ and Chi-Wai Kan * \\ Institute of Textile and Clothing, The Hong Kong Polytechnic University, Hung Hom, Kowloon, \\ Hong Kong SAR 999077, China; scarlett.yu@connect.polyu.hk (J.Y.); chenghao.lee@polyu.edu.hk (C.-H.L.) \\ * Correspondence: kan.chi.wai@polyu.edu.hk; Tel.: +852-2766-6531
}

Citation: Yu, J.; Lee, C.-H.; Kan, C.-W. Graphene Oxide/Reduced Graphene Oxide Enhanced Noniridescent Structural Colors Based on Silica Photonic Spray Paints with Improved Mechanical Robustness.

Nanomaterials 2021, 11, 949.

https: / doi.org/10.3390/nano11040949

\begin{abstract}
In contrast to traditional pigment colors, structural colors have developed a great potential in practical applications, thanks to their unique nonfading and color tunable properties; especially amorphous photonic structures with noniridescent structural colors have attracted considerable attention and their applications have expanded to more fields. Herein, graphene oxide (GO) and reduced graphene oxide (RGO) enhanced noniridescent structural colors with excellent mechanical robustness were established by a time-saving approach named spray coating, which allows for rapid fabrication of angular independent structural colors by spraying different photonic spray paints (PSPs) to ensure color multiplicity that was adjusted by the silica nanoparticles $\left(\mathrm{SiO}_{2} \mathrm{NPs}\right)$ sizes onto the substrates. The incorporation of poly(methyl methacrylate-butyl acrylate) (PMB) improved the adhesion existing among $\mathrm{SiO}_{2}$ inter-nanoparticles and between $\mathrm{SiO}_{2} \mathrm{NPs}$ and the substrates, taking advantages of the low glass transition temperature $\left(T_{g}\right)$ of butyl acrylate derivative polymer and made PMB embedded PSPs coated patterns being imparted with good mechanical robustness and abrasive resistance. The peculiar light adsorption of GO and RGO across visible light spectrum facilitate higher color saturation. The improvement in color saturation of GO and RGO doped PSPs is expected to boost the promising applications in structurally colored paintings, inks and other color-related optical fields.
\end{abstract}

Keywords: structural color; $\mathrm{SiO}_{2}$; nanoparticles; paints; graphene oxide; reduced graphene oxide; spray coating

\section{Introduction}

Noniridescent structural colors from amorphous photonic structures with short-range ordered arrangement based on incoherent light scattering [1-3], have attracted much scientific interest in recent years, owing to their superior non-fading, color tunable and angle-independent characteristics $[2,4]$. The materials with these superior optical performances and quasi-periodic, controlled disordered structures, namely photonic glasses or photonic alloys, have been proposed [5-7], different from photonic crystals that have longorder arranged structures by self-assembly [8,9]. Some fabrication approaches including electrophoretic deposition and dip coating, used to create structural-colored materials, seem to be delicate and time-consuming $[1,10,11]$. However, spray coating [12] is a relatively facile approach for rapid patterning structural colors especially angle-independent noniridescent structural colors as this requires minimal PSPs materials for spraying and is beneficial in mass production. Besides, it is widely accepted that $\mathrm{SiO}_{2} \mathrm{NPs}$ and its functionalized derivatives to form the building blocks for generating structural colours [13-17]. Kraft paper is a significant cellulose-based renewable material [18], its nonwoven porous structures with large surface areas, hydrophilicity, certain thickness and stiffness make it a
Copyright: (C) 2021 by the authors. Licensee MDPI, Basel, Switzerland. This article is an open access article distributed under the terms and conditions of the Creative Commons Attribution (CC BY) license (https:/ creativecommons.org/licenses/by/ $4.0 /)$. good candidate as the substrates for constructing structural colors.
Publisher's Note: MDPI stays neutral with regard to jurisdictional claims in published maps and institutional affiliations. 
The amorphous photonic structures with certain thicknesses possessing a strong incoherent light scattering in the visible light region results in whitish structural colors. In order to resolve this issue, black substances including carbon black [19-21], polydopamine [3,22] in small sizes and quantities have been used to suppress incoherent multiple scattering and improve the color visibility. Advanced carbon materials such as carbon nanoparticles, graphene oxide, graphene possessing exceptional mechanical, electrical, thermal and optical properties [23-27], contributing to the improvement of reflection color and visual appearance [28-30]. However, integration of these black materials with the structural color system has not been much studied. Moreover, since the structural stability of the photonic crystal or colloids film is poor due to the high $T_{g}$ of $\mathrm{SiO}_{2}$ and weak van der Waal's forces among the $\mathrm{SiO}_{2}$ NPs [31], and the fact that the black dopants cannot contribute to the mechanical robustness of structural color arrays, PMB was regarded as such an additive, benefiting from its relatively low $T_{g}$. The robust PSPs coated patterns are easily fabricated, which means that embedding $\mathrm{PMB}$ with $\mathrm{SiO}_{2} \mathrm{NPs}$ suspension is expected to endow the resulting PSPs coated films with strengthened adhesive performance and abrasion resisting properties.

In this paper, we present structural-colored PSPs on kraft paper substrates produced by spray coating that endows PSPs coated patterns with nearly angle-independent properties and noniridescent structural colors (Scheme 1). By controlling the $\mathrm{SiO}_{2} \mathrm{NPs}_{\text {sizes, certain }}$ ratios of dopants (PMB, GO, RGO) and thicknesses of sprayed PSPs coatings, diverse noniridescent structural colors can be easily realized. PSPs coated structurally colored materials with GO and RGO enhanced colors with excellent structural stability are expected to be desirable in broader applications in the fields of paintings and optical related areas.

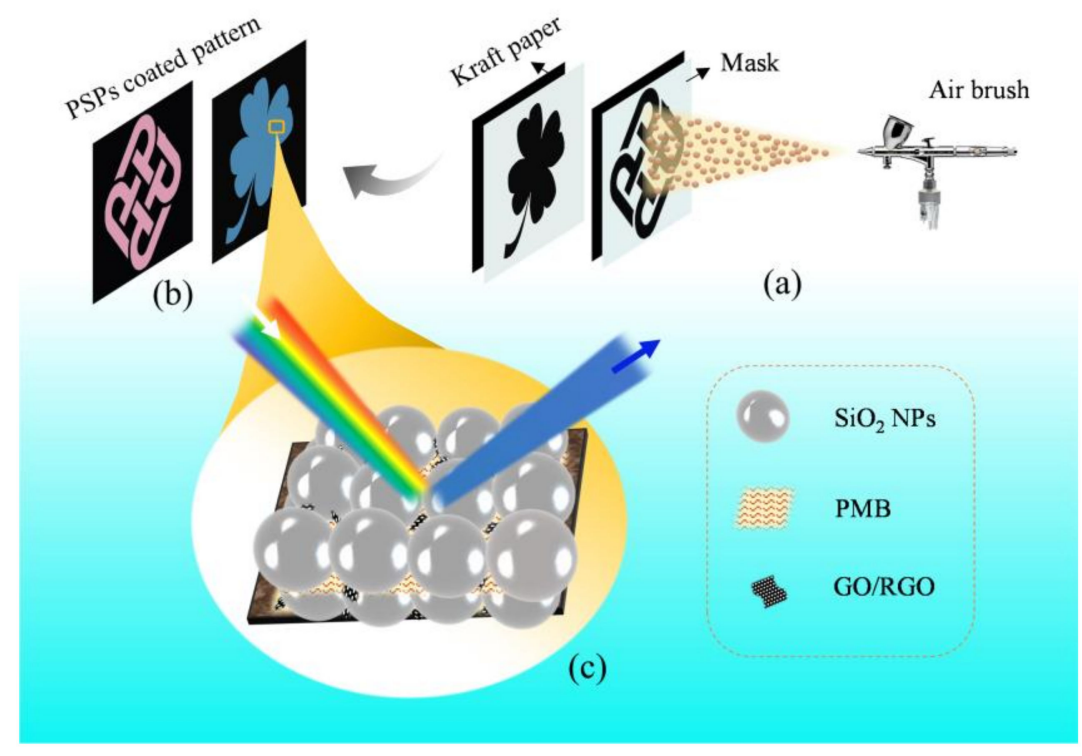

Scheme 1. (a) Schematic diagram of spray coating process of $\mathrm{SiO}_{2}$ PSPs on kraft paper using masks. (b) $\mathrm{SiO}_{2}$ PSPs coated patterns. (c) Model of $\mathrm{SiO}_{2}$ PSPs coated patterns with PMB dopants and GO or RGO enhanced noniridescent structural colors.

\section{Materials and Methods}

\subsection{Materials and Chemicals}

Tetraethyl orthosilicate (TEOS) (reagent grade), ammonia $\left(\mathrm{NH}_{3}, 25 \%\right.$ in $\left.\mathrm{H}_{2} \mathrm{O}\right)$ and $\mathrm{EtOH}, 99.9 \%$ ), sodium dodecyl sulfate (SDS), methyl methacrylate (MMA), butyl acrylate (BA, $\geq 99 \%$ ), ammonium persulfate (APS, $\geq 98.0 \%$, ACS reagent), graphene oxide ( $4 \mathrm{mg} \cdot \mathrm{mL}^{-1}$ dispersion in $\mathrm{H}_{2} \mathrm{O}$ ), reduced graphene oxide (powder) were purchased from Sigma-Aldrich (St. Louis, MO, USA). Deionised water was obtained from a Thermo Scientific GenPure Standard Water Purification System (Niederelbert, RP, Germany). GO and RGO were diluted and dispersed in deionized water by ultrasonication and formulated 
into $0.08 \mathrm{mg} \cdot \mathrm{mL}^{-1}$ aqueous solution before using. All chemicals were used as received from their venders without any further purification.

\subsection{Preparation of $P M B$}

To enhance the structural stability of the noniridescent structural colours, a kind of adhesive was synthesized by the following steps: $5.0 \mathrm{~g}$ MMA, $5.0 \mathrm{~g}$ BA, $1.0 \mathrm{~g}$ SDS, $25.0 \mathrm{~g}$ deionized water were sequentially added into a $100 \mathrm{~mL}$ double-deck three-necked flask in a water cycle bath that was controlled by Lab Companion RW3-0525 Refrigerated \& Heating Bath Circulator, with magnetic stirring at a speed of $500 \mathrm{rpm}$. After $30 \mathrm{~min}$ magnetic mixing at $80^{\circ} \mathrm{C}$, APS solution ( $0.1 \mathrm{~g}$ APS pre-dispersed in $5.0 \mathrm{~g}$ deionized water) was slowly added into the mixture. Afterwards, the mixture was stirred vigorously, and the reaction process was still kept at $80^{\circ} \mathrm{C}$ under a Nitrogen $\left(\mathrm{N}_{2}\right)$ atmosphere. The polymerization was carried out for $4 \mathrm{~h}$ and finally the weight ratio of the obtained PMB solution was $12.66 \%$.

\subsection{Preparation of $\mathrm{SiO}_{2}, \mathrm{SiO}_{2} / \mathrm{PMB}, \mathrm{SiO}_{2} / \mathrm{PMB} / \mathrm{GO}, \mathrm{SiO}_{2} / \mathrm{PMB} / \mathrm{RGO} P S P S$}

Various sizes of $\mathrm{SiO}_{2} \mathrm{NPs}$ were prepared based on the change of EtOH amount from 110 to $200 \mathrm{~mL}$. Deionized water $(15 \mathrm{~mL})$ and TEOS $(14 \mathrm{~mL})$ were added under magnetically stirring at $500 \mathrm{rpm}$ into a $250 \mathrm{~mL}$ double-deck three-necked flask equipped with a constant temperature water circulation system, followed by addition of $14 \mathrm{~mL}$ of ammonia water as a catalyst. After two-hour reaction process at a constant temperature of $60{ }^{\circ} \mathrm{C}, \mathrm{SiO}_{2} \mathrm{NPs}$ in suspension with different sizes were achieved by adjusting the amount of EtOH. The synthesized suspension solution of $\mathrm{SiO}_{2} \mathrm{NPs}$ was centrifuged and purified by centrifugation at $6000 \mathrm{rpm}$ for $20 \mathrm{~min}$. Then the supernatant solution was discarded, $\mathrm{SiO}_{2}$ NPs were dispersed and cleaned by deionized water. The purification step was repeated three times to obtain pure $\mathrm{SiO}_{2} \mathrm{NPs}$. $\mathrm{SiO}_{2}$ PSPs was obtained by redispersing the purified $\mathrm{SiO}_{2} \mathrm{NPs}$ into deionized water with $10 \%$ mass fraction. PMB $(5.0 \%$ to $\mathrm{SiO}_{2} \mathrm{NPs}$ ) was added into $10 \% \mathrm{SiO}_{2} \mathrm{NPs}$ stock solutions, and thus $\mathrm{SiO}_{2} / \mathrm{PMB}$ PSPs was obtained. In order to explore the GO or RGO effects on structural colors, GO or RGO was mixed with $\mathrm{SiO}_{2} / \mathrm{PMB}$ suspension (5.0\% $\mathrm{PMB}$ to $\mathrm{SiO}_{2} \mathrm{NPs}$ ) by ultrasonication to prepare $\mathrm{SiO}_{2} / \mathrm{PMB} / \mathrm{GO}, \mathrm{SiO}_{2} / \mathrm{PMB} / \mathrm{RGO}$ PSPs. Considering the relatively optimal brightness and saturation, $\mathrm{GO}$ or $\mathrm{RGO}\left(0.01 \%\right.$ to $\left.\mathrm{SiO}_{2} \mathrm{NPs}\right)$ was added in subsequent experiments.

\subsection{Fabrication of Noniridescent Structural Colors by Spray Coating}

PSPs coated patterns with noniridescent structural colors were facilely established by spray coating process, $\mathrm{SiO}_{2} \mathrm{PSPs}, \mathrm{SiO}_{2} / \mathrm{PMB}$ PSPs, $\mathrm{SiO}_{2} / \mathrm{PMB} / \mathrm{GO}$ PSPs or $\mathrm{SiO}_{2} / \mathrm{PMB} / \mathrm{RGO}$ PSPs were sprayed onto kraft paper through hollowed-out patterned polypropylene (PP) masks with continuous spraying every $20 \mathrm{~s}$. Every spaying lasting $10 \mathrm{~s}$ was regarded as one layer of PSPs on kraft paper. The distance between the nozzle of air brush and the masks in close proximity to kraft paper substrates was $10 \mathrm{~cm}$. To study $\mathrm{SiO}_{2} \mathrm{NPs}$ size change with EtOH and clearly observe PMB effect on the structural stability, $\mathrm{SiO}_{2}$ photonic crystal films (PCFs) were obtained by following steps: The stock solution of $10 \% \mathrm{SiO}_{2} \mathrm{NPs}$ and PMB (1-10\% to $\mathrm{SiO}_{2} \mathrm{NPs}$ ) were used for preparing $\mathrm{SiO}_{2} \mathrm{PCFs}$, a droplet of $400 \mu$ was then dropped on preheated cover glasses, followed by $50 \mathrm{~min}$ solvents evaporation at $65{ }^{\circ} \mathrm{C}$ in an oven, and finally $\mathrm{SiO}_{2}$ PCFs with iridescent colors and angle-dependent property were obtained.

\subsection{Characterization}

\subsubsection{Microscopic Observation}

The microstructure of PMB was detected by JEOL JEM-2011 transmission electron microscope (TEM, JEOL Co., Tokyo, Japan). PMB solution was first diluted by approximately 100 times, 10 nanoparticle solution was then deposited on 400-mesh carbon-coated copper grids, and the sample was completely air-dried prior to TEM observation. Scanning electron microscopy (SEM) images showing surface morphology of the prepared $\mathrm{SiO}_{2}$ PCFs with ordered hexagonal-close-packed structures were observed by VEGA3 TESCAN, 
imaging up to $80,000 \times$, and the micro-images of the PSPs coated patterns with disordered surfaces were performed by JSM-6490 SEM (JEOL Co., Tokyo, Japan). Prior to SEM observation, the non-conductive samples were sputter-coated with a thin gold film to reduce charge effects under micro imaging. The elemental analysis of the prepared samples was achieved by JSM-6490 SEM with an energy-dispersive X-ray spectroscopy (EDS) detector. The scanning probe characterization upon the morphological changes in the surface of $\mathrm{SiO}_{2}$ PCFs was analyzed by means of atomic force microscope (AFM) technique (Park systems, XE-100). The scan sizes $X$ and $Y$ were $2 \mu \mathrm{m}$ with $1.5 \mathrm{Z}$ servo grain at $0.8 \sim 1 \mathrm{~Hz}$ scan rate.

\subsection{2. $\mathrm{SiO}_{2} \mathrm{NPs}$ Sizes Measurement}

The average particle sizes and polydispersity index (Đ) of the as-synthesized $\mathrm{SiO}_{2}$ NPs were measured by a Zeta Plus particle size analyzer (Brookhaven Instruments Corp., Holtsville, NY, USA). The particle sizes of dried $\mathrm{SiO}_{2}$ NPs were calculated from TEM images by using Nano measurer software, version 1.2, Fudan University, Shanghai, China.

\subsubsection{Color Appearance Assessment}

Color reflectance and CIE $L^{*} a^{*} b^{*}$ values of the tested samples were analyzed by an SF 650 spectrophotometer (DataColor International, Lawrenceville, NJ, USA) with the D65 diffuse illumination, $8^{\circ}$ observing angle and $3 \mathrm{~mm} / 6.6 \mathrm{~mm}$ aperture sizes. Wavelengths ranging from 400 to $700 \mathrm{~nm}$ were obtained. Each datum was acquired by calculating the average value of four repeated measurements. CIE X and $\mathrm{Y}$ coordinates were calculated by ColorTell tool (ColorTell Tech Co., Ltd., Beijing, China) from $L^{*} a^{*} b^{*}$ values that was determined by SF 650 spectrophotometer. Optical images of the structural colour films were captured by a cellular dual $12 \mathrm{MP}$ wide-angle and telephoto cameras under the D65 illuminated light source in which the direction of illumination light is perpendicular to the ceiling of the chamber, and the color reflection images of the samples were obtained at the viewing angle $(\theta)$, between the sight line of the camera and the film sample surface. Besides, the light source cabinet was under totally dark environment.

\subsubsection{Spectroscopic Measurement}

The vibrational spectra of $\mathrm{PMB}, \mathrm{GO}, \mathrm{RGO}$ and PSPs including $\mathrm{SiO}_{2}, \mathrm{SiO}_{2} / \mathrm{PMB}$, $\mathrm{SiO}_{2} / \mathrm{PMB} / \mathrm{GO}$ and $\mathrm{SiO}_{2} / \mathrm{PMB} / \mathrm{RGO}$ were investigated by FTIR and Raman spectroscopy for rapid discrimination of chemical compositions of the prepared samples. FTIR spectrum with absorbance peak was obtained using a Perkin-Elmer Spectrum 100 FTIR spectrometer (Perkin-Elmer Inc., Boston, MA, USA) to analyze the inherent chemical information of PMB, $\mathrm{SiO}_{2}, \mathrm{GO}, \mathrm{RGO}, \mathrm{SiO}_{2} / \mathrm{PMB}, \mathrm{SiO}_{2} / \mathrm{PMB} / \mathrm{GO}$ and $\mathrm{SiO}_{2} / \mathrm{PMB} / \mathrm{RGO}$ PSPs. The samples were scanned from 4000 to $650 \mathrm{~cm}^{-1}$ region for 64 scans with a resolution of $4 \mathrm{~cm}^{-1}$ at room temperature. Micro-Raman spectrum was measured by Renishaw InVia Micro-Raman Spectroscopy system (Renishaw plc, Gloucestershire, UK) equipped with a confocal optical microscope for fast mapping. Raman wavelength range was between 200 and $2200 \mathrm{~nm}$, the spectral data was collected with resolution of $0.3 \mathrm{~cm}^{-1}$, and the stability was $< \pm 0.001 \mathrm{~cm}^{-1}$. The selected wavenumber ranged from 3200 to $300 \mathrm{~cm}^{-1}$, while the tested samples were irradiated with diode laser with excitation wavelength of $785 \mathrm{~nm}$, and the output power of laser was $0.01-5 \mathrm{~mW}$, adjusted by different samples. Raman scatterings from the samples were collected by objective lens with $50 \times$ eyepiece for visual observations. A multichannel charge-coupled device (CCD) detector was applied for spectral analyses, and a digital camera with high sensitivity mode was applied for imaging.

\subsubsection{Abrasion Test for Mechanical Robustness}

The sandpaper abrasion measurement was implemented by adding a $500 \mathrm{~g}$ weight on the back surface of a P100 sandpaper that was put face down on the tested samples that were fixed on a flat surface, pulling one end of the sandpaper for $1 \mathrm{~s}$, which leads to $5 \mathrm{~cm}$ movement of the weight along with sandpaper sliding over the sample surface. The sandpaper abrasion test was conducted twice for each sample. 


\section{Results and Discussion}

\subsection{Structural Colors Diversity Based on $\mathrm{SiO}_{2} \mathrm{NPs}$ Sizes}

The inverse correlation between $\mathrm{SiO}_{2}$ NPs size and the amount of ethanol (EtOH) is revealed intuitively (Figure 1a). Particle sizes of $\mathrm{SiO}_{2} \mathrm{NPs}$ ranging from $294.0 \mathrm{~nm}$ to $182.0 \mathrm{~nm}$ were obtained by regulating the amount of EtOH from $110 \mathrm{~mL}$ to $200 \mathrm{~mL}$. This study shows the size of the $\mathrm{SiO}_{2} \mathrm{NPs}$ decreases with the increase of $\mathrm{EtOH}$ volume while the amount of TEOS $(16 \mathrm{~mL})$, water $(15 \mathrm{~mL})$ and ammonia water $(14 \mathrm{~mL})$ remain fixed. The reflectance spectrum of the fabricated $\mathrm{SiO}_{2} \mathrm{PCF}$ varying from $\mathrm{SiO}_{2} \mathrm{NPs}$ sizes exhibiting iridescent structural colors on glasses shows conspicuous photonic bandgap displacement and proves a wide range of structural colors across the visible light region from $400 \mathrm{~nm}$ to $700 \mathrm{~nm}$ (Figure 1b). As the $\mathrm{SiO}_{2}$ NPs sizes decrease, the related wavelength of the photonic bandgap has a distinct blue shift. The result is consistent with the theoretical basis of Bragg's law Equation (1) [32,33], in which the characteristic peak wavelength of diffraction $(\lambda)$ is directly proportional to the interplanar spacing $(d)$ that is closely correlated to the sizes of self-assembled $\mathrm{SiO}_{2} \mathrm{NPs}$,

$$
m \lambda=2 d \sqrt{n_{e f f^{2}-\sin _{\varphi}^{2}}}
$$

where $m$ represents the series of diffraction, $n_{\text {eff }}$ means the effective refractive index, $\varphi$ is the angle of the incident light. It is also indicated that the structural color varies with the angle of incident light, resulting in angle-dependent structural colors in terms of $\mathrm{SiO}_{2}$ PCFs.
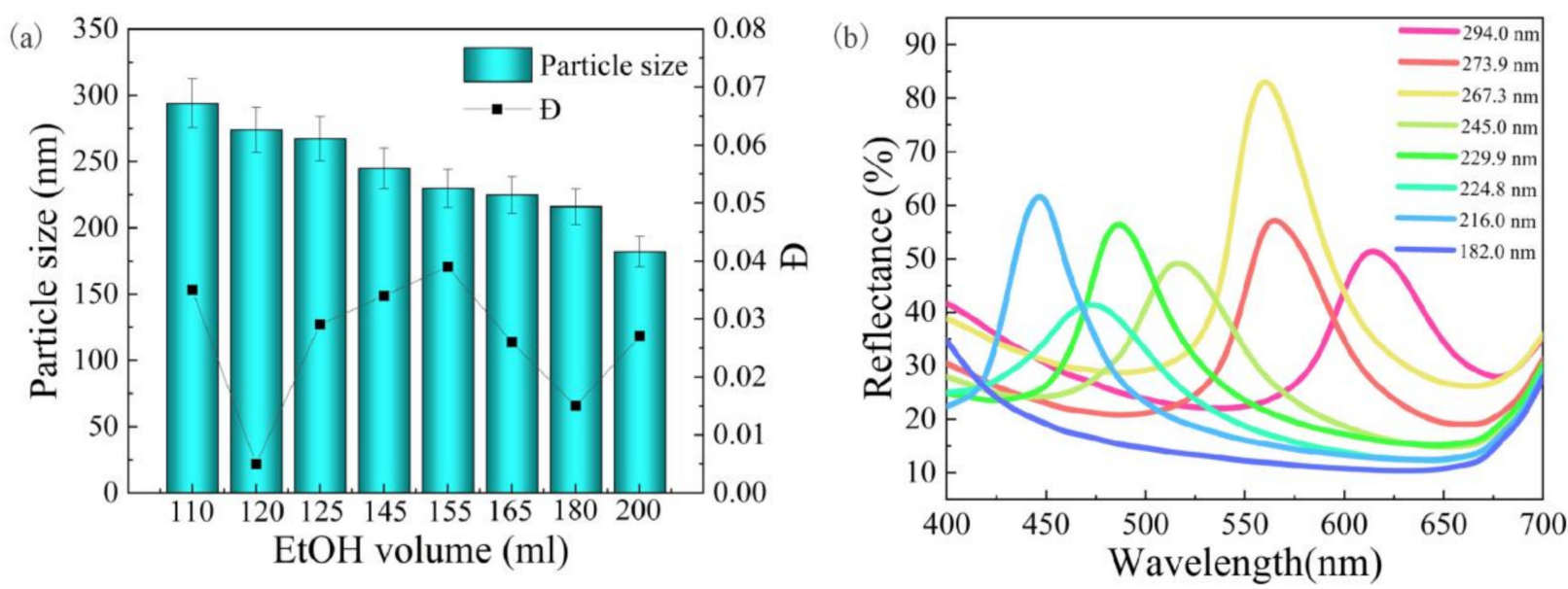

Figure 1. (a) Different mean $\mathrm{SiO}_{2}$ NPs sizes: 294.0, 273.9, 267.3, 245.0, 229.9, 224.8, 216.0 and $182.0 \mathrm{~nm}$ with different $Đ$ values as a function of EtOH amount: 100, 120, 125, 145, 155, 165, 180 and $200 \mathrm{~mL}$ respectively. (b) The reflectance spectra of $\mathrm{SiO}_{2}$ PCFs that were constructed by self-assembled $\mathrm{SiO}_{2} \mathrm{NPs}$ with controllable sizes in visible light region.

It was found from SEM observation that $\mathrm{SiO}_{2} \mathrm{NPs}$ with the spherical shapes and relatively uniform diameters were arranged in hexagonal close packed structures periodically. These honeycomb like crystalline structures of $\mathrm{SiO}_{2} \mathrm{PCFs}$ were found in the area of yellow boxes as shown in Figure 2a1-h1. The $\mathrm{SiO}_{2}$ NPs sizes decreased apparently with the increase of EtOH volume from both SEM (Figure 2a1-h1) and AFM images (Figure 2a3-h3). It coincided well with experimental results as illustrated in Figure 1a. Histograms (Figure 2a2-h2) were used to intuitively analyse $\mathrm{SiO}_{2}$ particle size distribution with different average diameters measured from SEM images by Nano Measurer software, the average $\mathrm{SiO}_{2}$ NPs sizes were calculated after measurement of the diameters of 100 selected $\mathrm{SiO}_{2} \mathrm{NPs}$ from the SEM micrographs. It was showed that diameters of each kind of $\mathrm{SiO}_{2} \mathrm{NPs}$ were normally distributed and comparatively centralized in certain average particle sizes. There was a difference in particle size results measured by Zeta Plus particle size analyser and Nano Measurer software. It was showed that numerical difference of $\mathrm{SiO}_{2}$ 
NPs sizes obtained by Nano Measurer from SEM images was more than $10 \mathrm{~nm}$ smaller than that measured by Zeta Plus particle size analyzer. The reason behind this phenomenon could be attributed to the different $\mathrm{SiO}_{2} \mathrm{NPs}$ states based on different test conditions, in which Brownian dynamics of $\mathrm{SiO}_{2} \mathrm{NPs}$ existed in diluted aqueous suspension that was used for particle size analysis by Zeta Plus analyzer, while dried $\mathrm{SiO}_{2} \mathrm{NPs}_{\text {sith removal }}$ of aqueous media can be regarded as SEM samples, the solvent evaporation shielded $\mathrm{SiO}_{2} \mathrm{NPs}$ size measurement from the effect of Brownian hydrodynamic interactions, and thus results in smaller nanoparticle sizes. From AFM observation (Figure 2a3-h3), the measurements on the topography of these $\mathrm{SiO}_{2} \mathrm{NPs}$ further confirmed the occurrence of the expected arrangement consisting of hexagonal arrays on the surface of the $\mathrm{SiO}_{2} \mathrm{PCF}$ with few defects, and there was a manifest decrease in particle sizes with $\mathrm{EtOH}$ volume increase in accordance with the SEM results. Particle sizes of each sample were estimated to be uniform and had dimensional homogeneity. Moreover, the spherical integrity has been confirmed, however, it is interesting that the shape of $\mathrm{SiO}_{2} \mathrm{NPs}$ seems to be rugby-like (Figure 2b4), the distortion of the particle shape in AFM images is probably ascribed to image signals derived from the geometrical interaction between the particle surface and the finite size pyramidal-shaped tip. TEM results proved that $\mathrm{SiO}_{2} \mathrm{NPs}$ are in spherical shapes (Figure S1).
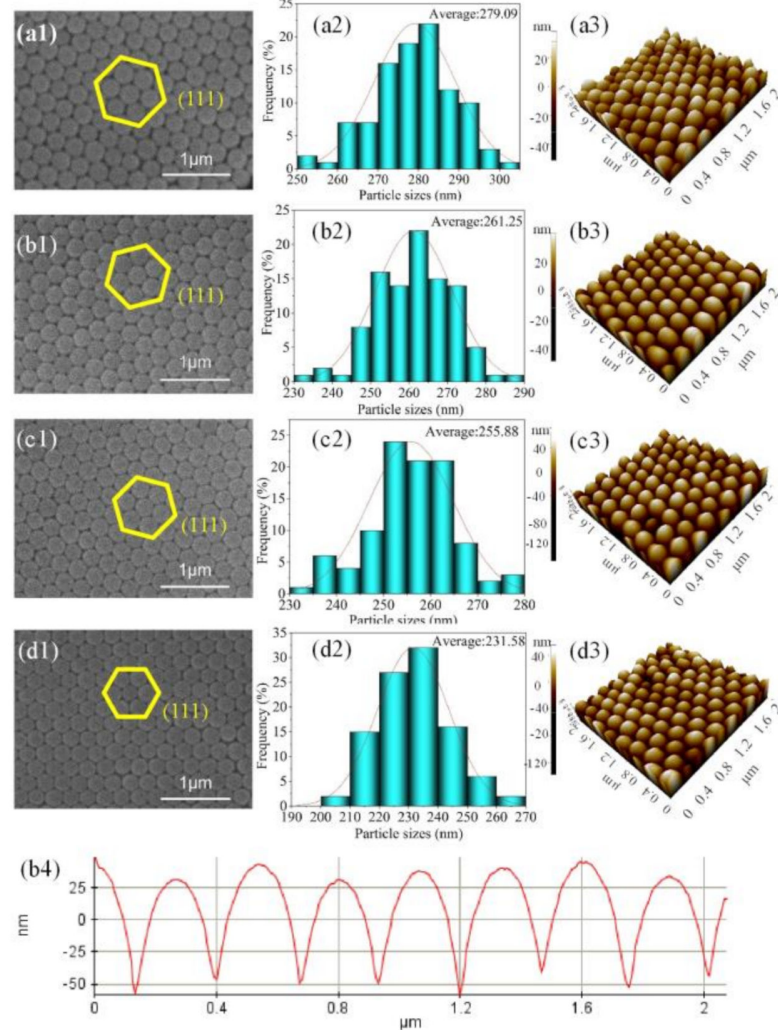
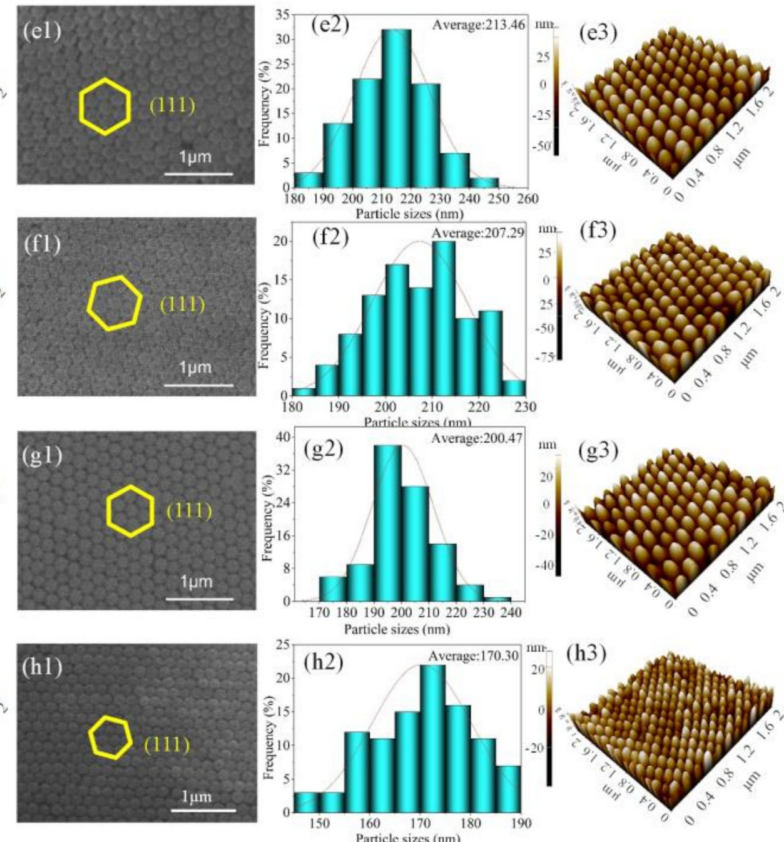

Figure 2. $\mathrm{SiO}_{2}$ PCFs with $\mathrm{SiO}_{2} \mathrm{NPs}$ sizes (a) $294.0 \mathrm{~nm}$, (b) $273.9 \mathrm{~nm}$, (c) $267.3 \mathrm{~nm}$, (d) $245.0 \mathrm{~nm}$, (e) $229.9 \mathrm{~nm}$, (f) 224.8 nm, (g) $216.0 \mathrm{~nm}$, (h) $182.0 \mathrm{~nm}$ measured by Zeta Plus particle size analyser. (a1-h1) SEM micrographs of $\mathrm{SiO}_{2} \mathrm{PCFs}_{\text {with }}$ honeycomb structures as shown in the areas of yellow frames (magnification: $80,000 \times$ ). (a2-h2) histogram of corresponding particle size distribution with different average diameters measured by Nano Measurer software: $279.09 \mathrm{~nm}, 261.25 \mathrm{~nm}$, $255.88 \mathrm{~nm}, 231.58 \mathrm{~nm}, 213.46 \mathrm{~nm}, 207.29 \mathrm{~nm}, 200.47 \mathrm{~nm}, 170.30 \mathrm{~nm}$. The red lines were the curves obtained by Gaussian fitting. (a3-h3) Three-dimensional (3-D) representations of surface morphology of $\mathrm{SiO}_{2} \mathrm{NPs}$ measured by AFM showing within close-packed hexagonal cone structures. The measured area is $2 \times 2 \mu \mathrm{m}^{2}$. (b4) Section analysis of $\mathrm{SiO}_{2} \mathrm{NPs}(273.9$ $\mathrm{nm}$ ) by atomic force microscopy (AFM). 


\subsection{Noniridescent Structural Colours by Spray Coating}

Color properties of $\mathrm{SiO}_{2}$ PSPs coated patterns exhibiting noniridescent structural colors by spray coating method was evaluated (Figure 3). The PSPs coated patterns with 1-10 spraying layers ( $\mathrm{L} 1$ to L10) were selected as the research objects, in which the selected $\mathrm{SiO}_{2} \mathrm{NPs}$ is $245 \mathrm{~nm}$ (Figure 3b). Spraying 1 or 2 layers of PSPs on the substrates exhibited gloomy and muted structural colors. After spraying reached 4 layers (L4), the structural color tends to be clearer; the unevenness of the structural color is attributed to the shaking of air brush during spray coating process. However, as the spraying layers reached 7 or 10 layers, the structural colors seemed to be whitish. Numerical analysis on reflectance of $\mathrm{SiO}_{2}$ PSPs with diverse thickness is shown in Figure 3d. The reflectance peak of L1 and L2 is lower (around 10\%) due to the incomplete coverage of $\mathrm{SiO}_{2}$ PSPs on black substrate, resulting in absorbance of scattering. As the spraying layers increased to $\mathrm{L} 4$, the reflectance peak increased (around 20\%), and after further spraying, the peak reflectance reached $28 \%$ and $45 \%$, respectively. The results correspond to the observation by naked eye (Figure $3 b$ ). We selected PSPs coated patterns with 4 spraying layers (L4) as the following objects to study the angle-independence property of $\mathrm{SiO}_{2}$ PSPs coated patterns (Figure 3c,e-g). The observation method is displayed schematically in Figure $3 a$, in which the viewing angle is $\theta$. Figure $3 c$ proves the slightly angle-dependent property of PSPs coated patterns exhibiting diverse noniridescent structural colours at $30^{\circ}, 45^{\circ}, 60^{\circ}$ and $90^{\circ}$ and the structural colours were hardly changed with the change of viewing angle. The reflectance spectra of these patterns with four distinct structural colors (Figure 3f). It is noticeable that broader photonic bandgap based on coherent reflection is yielded by photonic amorphous structures in shortrange order of PSPs coated patterns by spray coating, substantially different from the narrow one in photonic crystalline arrays of $\mathrm{SiO}_{2} \mathrm{PCFs}$ (Figures 1 and 2). While the sizes of $\mathrm{SiO}_{2} \mathrm{NPs}$ decreased from $294.0 \mathrm{~nm}, 267.3 \mathrm{~nm}, 245.0 \mathrm{~nm}$ to $229.9 \mathrm{~nm}$, the photonic bandgap peak wavelength had a similar synchronized decline from 610, 590, 510 to $480 \mathrm{~nm}$. For more visualized observation, these four structural colors were converted into Commission Internationale de $\mathrm{l}^{\prime}$ Eclairage (CIE) chromaticity coordinates (Figure 3g). 

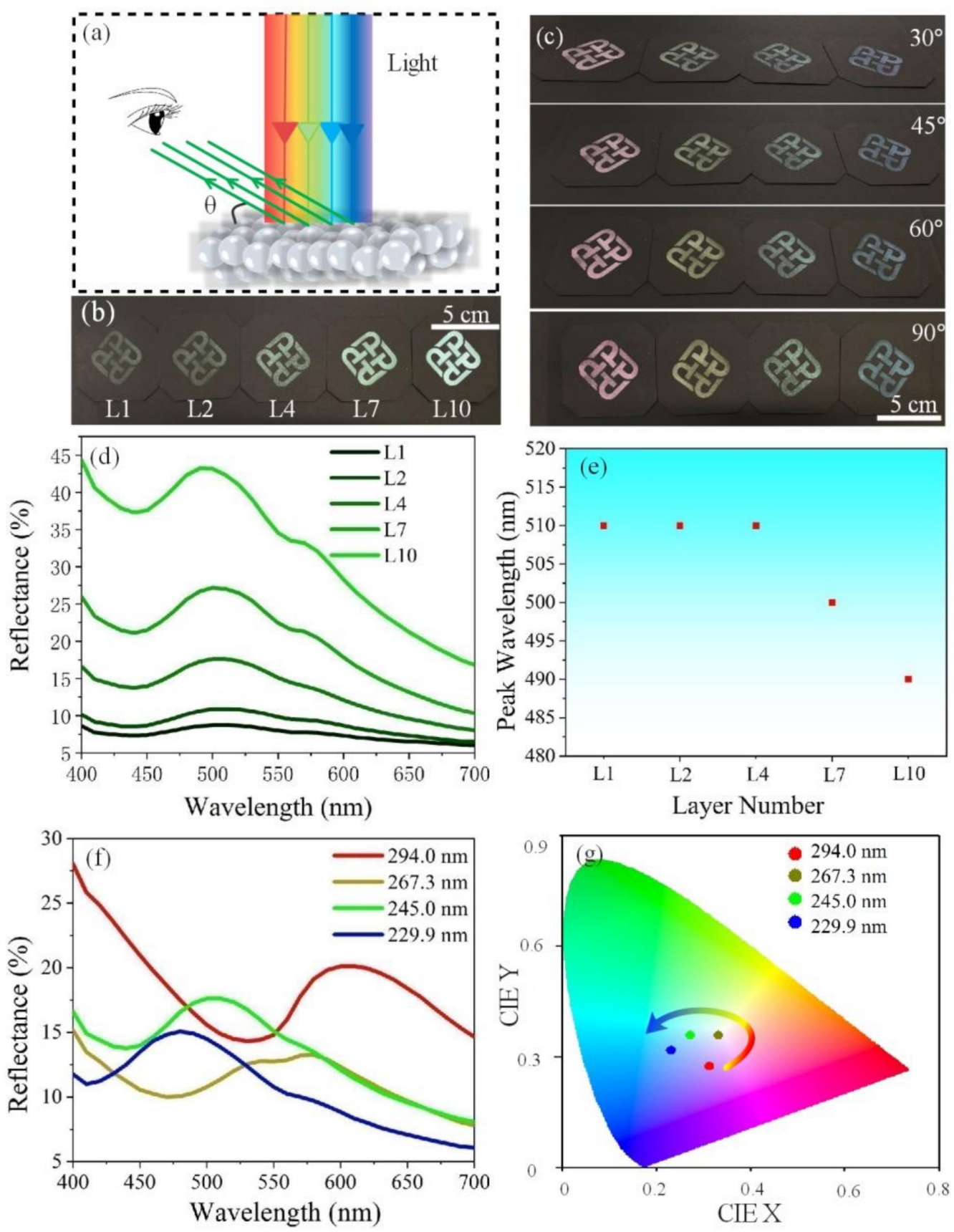

Figure 3. (a) Schematic representation of structural color observation with viewing angle $(\theta)$. (b) Photographs of $\mathrm{SiO}_{2}$ PSPs $(245 \mathrm{~nm})$ coated PolyU logo patterns with different thicknesses of PSPs (various spraying layers) from 1 to 10 (L1 to L10). (c) Photographs of PolyU logo patterns with four structural colors using $\mathrm{SiO}_{2}$ PSPs with different $\mathrm{SiO}_{2} \mathrm{NPs}$ sizes from $294.0 \mathrm{~nm}, 267.3 \mathrm{~nm}$, $245.0 \mathrm{~nm}$ to $229.9 \mathrm{~nm}$ at different viewing angles $\left(30^{\circ}, 45^{\circ}, 60^{\circ}, 90^{\circ}\right)$. (d,e) Reflectance spectra and the corresponding maximum reflection peak wavelength of PSPs coated patterns in (b). (f) Reflectance spectra of the four structural colors in (c). (g) The CIE chromaticity diagram of the four structural colors in (c).

\subsection{Mechanical Robustness Enhancement}

Due to the pure $\mathrm{SiO}_{2}$ PCFs or PSPs suffering from unstable structural colours and poor mechanical strength with the substrates, PMB was introduced as a kind of binder to improve structural stability. To manifestly investigate the adhesion function of PMB on $\mathrm{SiO}_{2} \mathrm{PCFs}$, photonic crystal films with structural colors were fabricated on glass slide 
substrates with smooth and frictionless surfaces. The structures of PMB copolymer were displayed as the TEM observation from Figure 4a,b. Poly(butyl acrylate) occupied the positions between the adjacent $\mathrm{SiO}_{2} \mathrm{NPs}$ and provided the essential adhesion among them. Differential scanning calorimetry (DSC) result indicated low $T_{g}$ (around $-50{ }^{\circ} \mathrm{C}$ ) of PBA and it showed the low glass transition temperature around $-50{ }^{\circ} \mathrm{C}$ to $0{ }^{\circ} \mathrm{C}$, indicating good elasticity of PMB. The existence of PMMA was also proved because $T_{g}$ around $95^{\circ} \mathrm{C}$ was associated with PMMA (Figure S2). Brilliant structural colours (Figure 4c) were generated from long-ordered crystalline structures of $\mathrm{SiO}_{2}$ PCFs owing to the narrow and sharp photonic bandgap (Figure 1b). It can be obviously seen from Figure $4 \mathrm{c}$ that bare $\mathrm{SiO}_{2} \mathrm{PCFs}$ was fragile and easily peeled off from the substrate due to its high $T_{g}$, and this phenomenon is also ascribed to the weak inter-interactions among $\mathrm{SiO}_{2}$ photonic crystal building blocks as well as interactions between $\mathrm{SiO}_{2}$ PCFs and the substrate [34]. With the increase of PMB amount, there were hardly any cracks and disintegrating slags of $\mathrm{SiO}_{2} \mathrm{PCF}$ which confirms that PMB made $\mathrm{SiO}_{2}$ PCFs that made it having mechanically robust and it was strongly bonded to the substrates, thanks to the extremely low $T_{g}$ of the polymer synthesised by BA monomer [35]. Nonetheless, too much PMB (7.5\% and 10.0\%) resulted in many cracks of $\mathrm{SiO}_{2}$ PCFs because of PMB being the obstacle in self-assembly of $\mathrm{SiO}_{2} \mathrm{NPs}$ with the removal of solvent by evaporation. It is noticed that the structural colors have an obvious red shift with the increase of PMB content (Figure 4c), which is verified by the reflectance in Figure 4d. Evidently, the primary peak wavelength is enlarged from $500 \mathrm{~nm}, 510$ to $520 \mathrm{~nm}$ as the PMB content increased from $1.0 \%$ to $10.0 \%$, and it represents the red shift of color (Figure 4e). Interestingly, reflectance of bare $\mathrm{SiO}_{2} \mathrm{PCFs}$ shows a single peak, however, double peak of reflectance appears with the addition of PMB (Figure 4d,e). Taking $\mathrm{SiO}_{2}$ PCFs with 5.0\% PMB as an example, the corresponding structural color becomes yellowish on blending green (primary peak: $520 \mathrm{~nm}$ ) and reddish orange (secondary peak: $590 \mathrm{~nm}$ ). It can be explained that the inclusion of PMB changed the effective refractive index $(n)$ in photonic crystal lattice as illustrated in Equation (1).
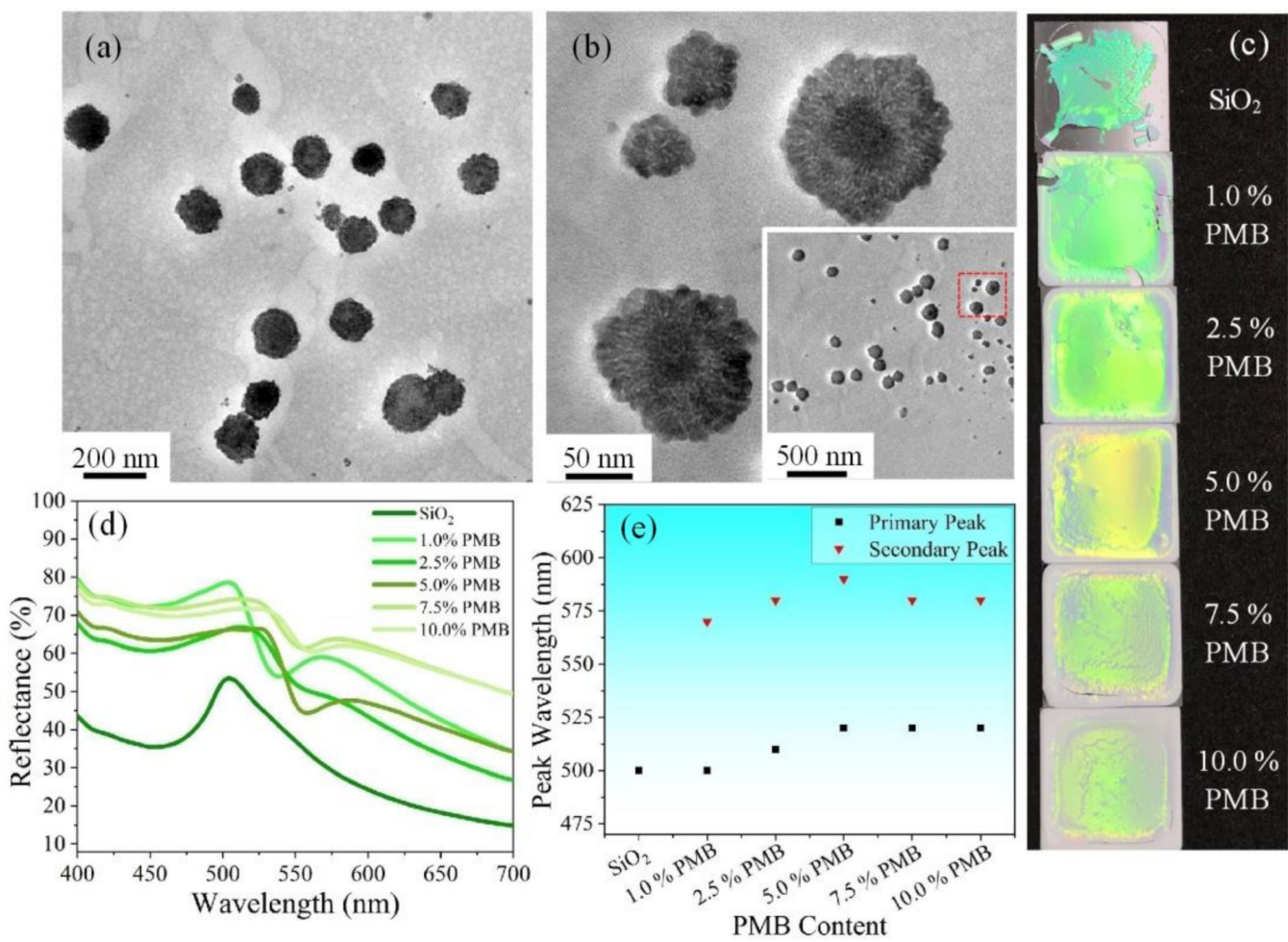

Figure 4. TEM images of poly(methyl methacrylate-butyl acrylate) (PMB) with magnification of (a) $12,000 \times$, (b) 50,000 $\times$, inserted zoom-out image: $8000 \times$. (c) Photos of $\mathrm{SiO}_{2}$ PCFs with different concentrations of PMB on glasses. (d) The related reflectance of (c). (e) The peak photonic bandgap wavelength of (d). 


\subsection{Inclusion of GO and RGO into PSPs Coated Patterns}

Since noniridescent structural colors prepared by spray coating method are relatively pale and whitish compared to those generated from PCFs due to the strong incoherent scattering that dilutes the light from photonic bandgap [36], black substances (GO or RGO) with light scattering absorbing ability are introduced in colloidal nanoparticle suspension to improve the color saturation and contrast. Figure $5 \mathrm{a} 1$,a2 shows the nanostructures of kraft paper having many openings, holes with large surface contact area, and intrinsic hydrophilicity of cellulosic component are conducive to infiltration of sprayed PSPs and rapid evaporation of water medium during spray coating process. Unlike the crystalline structures of PCFs (Figure 2), photonic amorphous structures existed in PSPs coated patterns created by spray coating as Figure $5 \mathrm{~b} 2-\mathrm{e} 2$ shows. Dielectric spherical nanoparticles can possess strong scattering resonances based on Mie theory [37]. Such disordered structures with no long-range order impede light interferences but increase the scattering effect, in which the Mie resonances in dielectric nanoparticles $\left(\mathrm{SiO}_{2} \mathrm{NPs}\right)$ with high refractive index in the several hundred-nanometer regions contribute to generate structural color $[33,38]$. Figure $5 \mathrm{~b} 1, \mathrm{~b} 2$ proves that pure $\mathrm{SiO}_{2}$ PSPs coated film is vulnerable to cracks on a large scale, while the crack-free surface and continuity of amorphous structures about $\mathrm{SiO}_{2} / \mathrm{PMB}$ PSPs coated films is convincing (Figure 5c1,c2), which corresponds to the investigation in Figure 4c. EDS analysis indicates the existence of $\mathrm{PMB}$ with the increase of $\mathrm{O}$ weight percent and the appearance of $\mathrm{C}$ element from (Figure $5 \mathrm{c} 3$ ). The inclusion of well-dispersed $\mathrm{GO}$ and RGO in small nanoscale did not alter the crack-free surface of $\mathrm{SiO}_{2} / \mathrm{PMB} / \mathrm{GO}$ and $\mathrm{SiO}_{2} / \mathrm{PMB} / \mathrm{RGO}$ coated patterns (Figure 5d2,e2). It can be seen from Figure $5 \mathrm{~d} 3$,e3 that the decrease of $\mathrm{O}$ weight ratio from $62.94 \%$ to $59.23 \%$ validates the fact that RGO has been reduced from GO before use.

The band profiles of $\mathrm{PMB}, \mathrm{GO}, \mathrm{RGO}$ and $\mathrm{SiO}_{2}, \mathrm{SiO}_{2} / \mathrm{PMB}, \mathrm{SiO}_{2} / \mathrm{PMB} /$ $\mathrm{GO}, \mathrm{SiO}_{2} / \mathrm{PMB} / \mathrm{RGO}$ PSPs were performed experimentally by Fourier transform infrared (FTIR) spectrum (Figure S3) and Raman spectra (Figure 6). In FTIR analysis, it is confirmed that the peak at $1731 \mathrm{~cm}^{-1}$ belongs to $\mathrm{C}=\mathrm{O}$ stretching vibration, while the characteristic peak at $1158 \mathrm{~cm}^{-1}$ is assigned to $\mathrm{C}-\mathrm{O}-\mathrm{C}$ stretching vibration of the MMA and $\mathrm{BA}$ repeating units [39]. The corresponding characteristic peak in transmittance spectra of $\mathrm{SiO}_{2} / \mathrm{PMB}$ PSPs shows PMB with small amounts existed in $\mathrm{SiO}_{2} / \mathrm{PMB}$ PSPs. From Figure S3c, broad band of GO transmittance spectra from 3500 to $2500 \mathrm{~cm}^{-1}$ refers to hydrogen bonds of carboxyl COOH groups [40], $3400 \mathrm{~cm}^{-1}$ is related to the stretching of hydroxyl group mainly as a result of the presence of absorbed water molecules and alcohol group [41], the peak at $1225 \mathrm{~cm}^{-1}$ is attributed to $\mathrm{C}-\mathrm{OH}$ stretching of alcohol group, $\mathrm{C}=\mathrm{O}$ carbonyl stretching appeared at $1718 \mathrm{~cm}^{-1}$ [42], $1053 \mathrm{~cm}^{-1}$ corresponds to $\mathrm{C}-\mathrm{O}$ group [43]. RGO was obtained by reduction of GO, and thus the related hydroxyl groups are hardly seen in the spectra of RGO; the RGO characteristic peaks are not obvious. The characteristic peaks of GO and RGO are also not apparent in the FTIR spectra of $\mathrm{SiO}_{2} / \mathrm{PMB} / \mathrm{GO}, \mathrm{SiO}_{2} / \mathrm{PMB} / \mathrm{RGO}$ PSPs owing to the addition of GO and RGO in small amounts (Figure S3d). To further demonstrate the presence of $\mathrm{GO}$ and $\mathrm{RGO}$ in $\mathrm{SiO}_{2} / \mathrm{PMB} / \mathrm{GO}, \mathrm{SiO}_{2} / \mathrm{PMB} / \mathrm{RGO}$ PSPs, Raman spectroscopy was performed on PMB, GO, RGO and $\mathrm{SiO}_{2}, \mathrm{SiO}_{2} / \mathrm{PMB}, \mathrm{SiO}_{2} / \mathrm{PMB} / \mathrm{GO}$, $\mathrm{SiO}_{2} / \mathrm{PMB} / \mathrm{RGO}$ PSPs to analyze the interface interaction between nanocomposites and structural changes, the main characteristic peaks of these samples are shown in Figure 6. Two prominent bands of GO at $1354 \mathrm{~cm}^{-1}$ (D band) and $1600 \mathrm{~cm}^{-1}$ (G band) shifted to $1314 \mathrm{~cm}^{-1}$ (D band) and $1597 \mathrm{~cm}^{-1}$ (G band) for RGO after reduction. The relative intensity ratio $\left(I_{D} / I_{G}\right)$ obviously increases from $\mathrm{GO}$ to $\mathrm{RGO}$, which indicates more defects generated for RGO after reduction reaction. Analogous experiment has been conducted by Sharma et al. [42]. Raman spectra of $\mathrm{SiO}_{2} / \mathrm{PMB} / \mathrm{GO}$ and $\mathrm{SiO}_{2} / \mathrm{PMB} / \mathrm{RGO}$ PSPs both show $\mathrm{G}$ band, indicating the existence of $\mathrm{GO}$ and $\mathrm{RGO}$, respectively. 


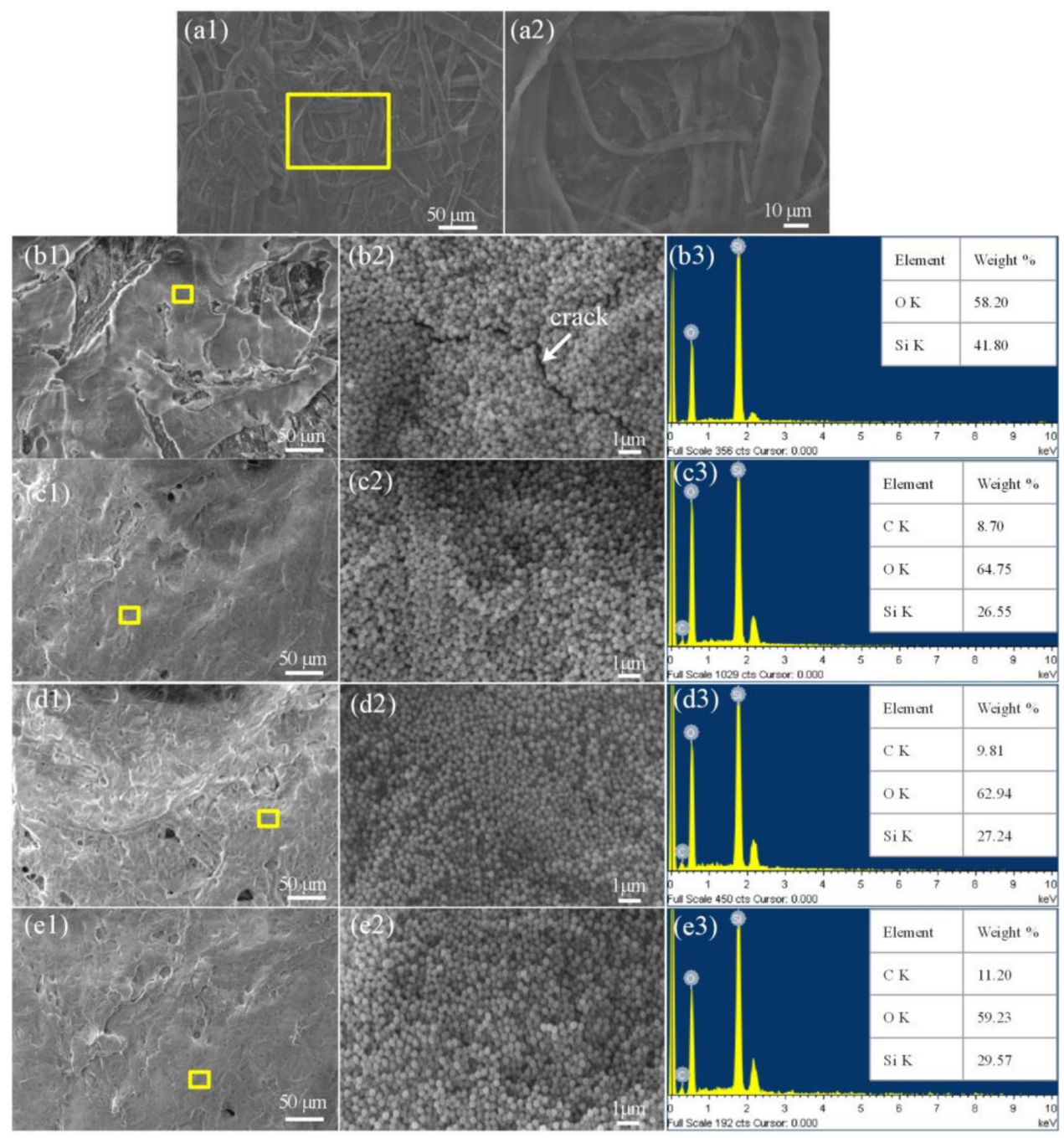

Figure 5. (a) Surface morphology of kraft paper from SEM observation with magnification of (a1) $300 \times$ and (a2) $1000 \times$. (b-e) $\mathrm{SiO}_{2}, \mathrm{SiO}_{2} / \mathrm{PMB}, \mathrm{SiO}_{2} / \mathrm{PMB} / \mathrm{GO}$ and $\mathrm{SiO}_{2} / \mathrm{PMB} / \mathrm{RGO} \mathrm{PSPs}$, respectively. (b1-e1,b2-e2) surface morphology with magnification of (b1-e1) 300× and (b2-e2) $10,000 \times$. (b2-e2) represents the zoom-in area of the yellow frame in (b1-e1). (b3-e3) Elemental analysis $(\mathrm{C}, \mathrm{O}, \mathrm{Si})$ of $\mathrm{SiO}_{2}, \mathrm{SiO}_{2} / \mathrm{PMB}, \mathrm{SiO}_{2} / \mathrm{PMB} / \mathrm{GO}$ and $\mathrm{SiO}_{2} / \mathrm{PMB} / \mathrm{RGO}$ PSPs. 


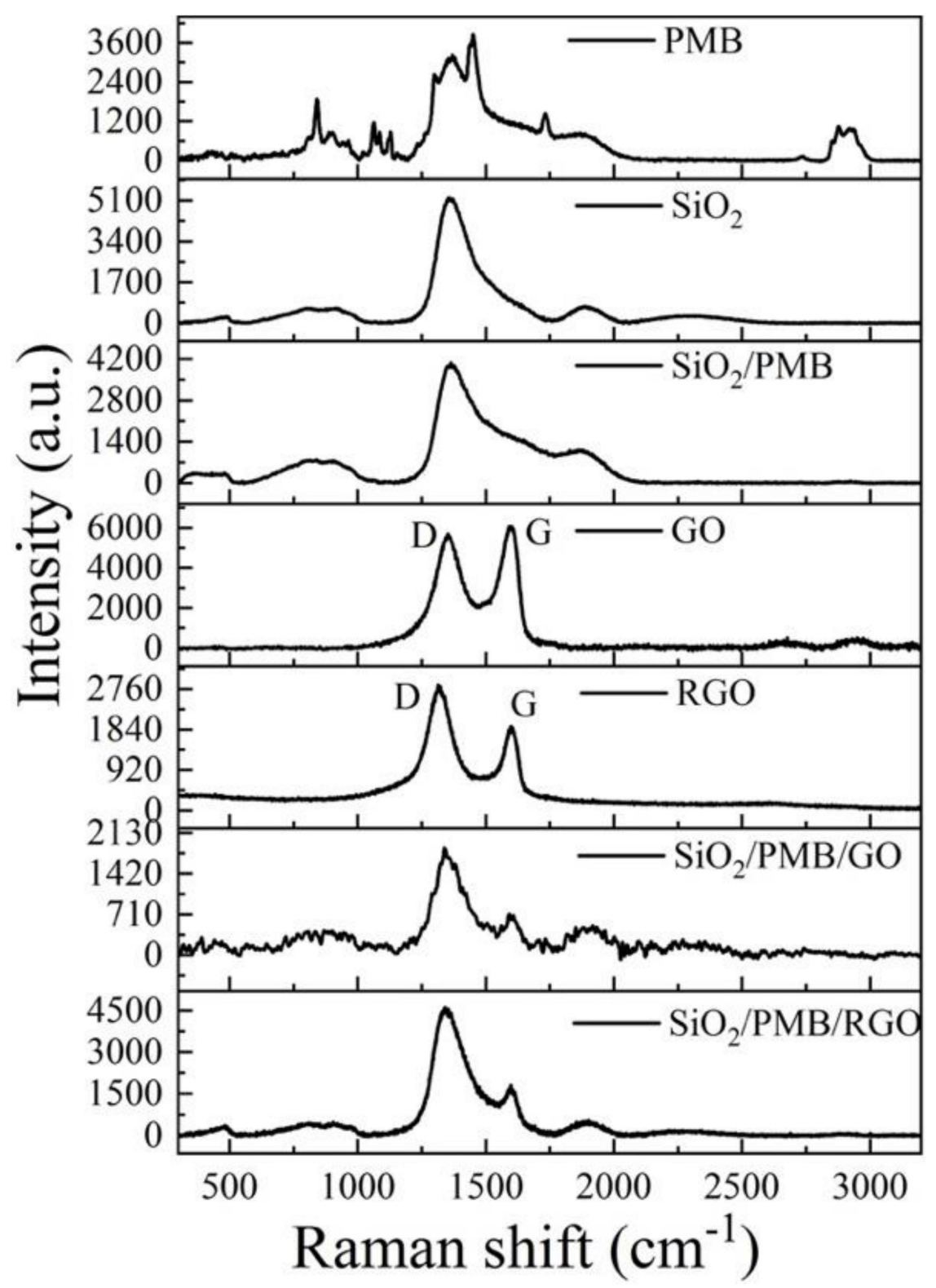

Figure 6. Raman spectrum of PMB, graphene oxide (GO), reduced graphene oxide (RGO) and photonic spray paints (PSPs) including $\mathrm{SiO}_{2}, \mathrm{SiO}_{2} / \mathrm{PMB}, \mathrm{SiO}_{2} / \mathrm{PMB} / \mathrm{GO}$ and $\mathrm{SiO}_{2} / \mathrm{PMB} / \mathrm{RGO}$.

\subsection{Mechanical Robustness Measurement}

Abrasion repellence is another area to expand the wide applications of the PSPs coated paintings for goods preservation. In order to verify whether mechanical robustness of PSPs coated patterns by spray coating is enhanced, we conducted an abrasion test for PSPs coated four-leaf clover patterns. $\mathrm{SiO}_{2}$ PSPs coated pattern produced color whitening problem (Figure $7 \mathrm{~b} 1$ ), however, color saturation was enhanced with inclusion of GO and RGO (Figure 7b3,b4). The corresponding reflectance (Figure 7d) confirms the decrease of reflectance peak after addition of GO and RGO, mainly thanks to these black dopants that improve the absorbance of light scattering. Upon application of mechanical forces, pure $\mathrm{SiO}_{2}$ PSPs coated patterns showed more defects and had more leakage of $\mathrm{SiO}_{2} \mathrm{NPs}$ after abrasion (Figure $7 \mathrm{~b}, \mathrm{c}$ ), which is consistent with the findings of $\mathrm{SiO}_{2} \mathrm{PCFs}$ (Figure 4). Moreover, as Figure 7d,e displays, the corresponding peak reflectance of $\mathrm{SiO}_{2} \mathrm{PSPs}$ coated patterns decreased sharply from $15.5 \%$ to $11.2 \%$ owing to the reveal of black kraft paper substrate, while other $\mathrm{SiO}_{2} / \mathrm{PMB}, \mathrm{SiO}_{2} / \mathrm{PMB} / \mathrm{GO}$ and $\mathrm{SiO}_{2} / \mathrm{PMB} / \mathrm{RGO}$ coated patterns maintained their structural colors with few defects under friction due to PMB. It is worth 
nothing that red shift also appeared in $\mathrm{SiO}_{2} / \mathrm{PMB}, \mathrm{SiO}_{2} / \mathrm{PMB} / \mathrm{GO}$ and $\mathrm{SiO}_{2} / \mathrm{PMB} / \mathrm{RGO}$ coated patterns (Figure $7 \mathrm{~b} 2-\mathrm{b} 4$ ) compared with pure $\mathrm{SiO}_{2}$ PSPs coated pattern (Figure $7 \mathrm{~b} 1$ ), and the structural colors collaboratively turned from bluish violet to dark yellowish green. The occurrence of red shift is mainly due to addition of PMB polymer adhesive that increased the effective refractive index [31], resulting in the increase of photonic bandgap wavelength. This result is in accord with the investigation that the structural colour change occurred in PCFs with and without PMB (Figure 4c). Correspondingly, the peak reflectance shifted from $460 \mathrm{~nm}\left(\mathrm{SiO}_{2} \mathrm{PSPs}\right)$ to $470 \mathrm{~nm}\left(\mathrm{SiO}_{2} / \mathrm{PMB}\right.$ PSPs), the peak reflectance of $\mathrm{SiO}_{2} / \mathrm{PMB} / \mathrm{GO}$ and $\mathrm{SiO}_{2} / \mathrm{PMB} / \mathrm{RGO}$ coated patterns had no significant change in contrast to $\mathrm{SiO}_{2} / \mathrm{PMB}$ PSPs coated one. Nevertheless, colour saturation of the structural colors was improved with the addition of GO and RGO as can be seen from Figure 7b1-b4.
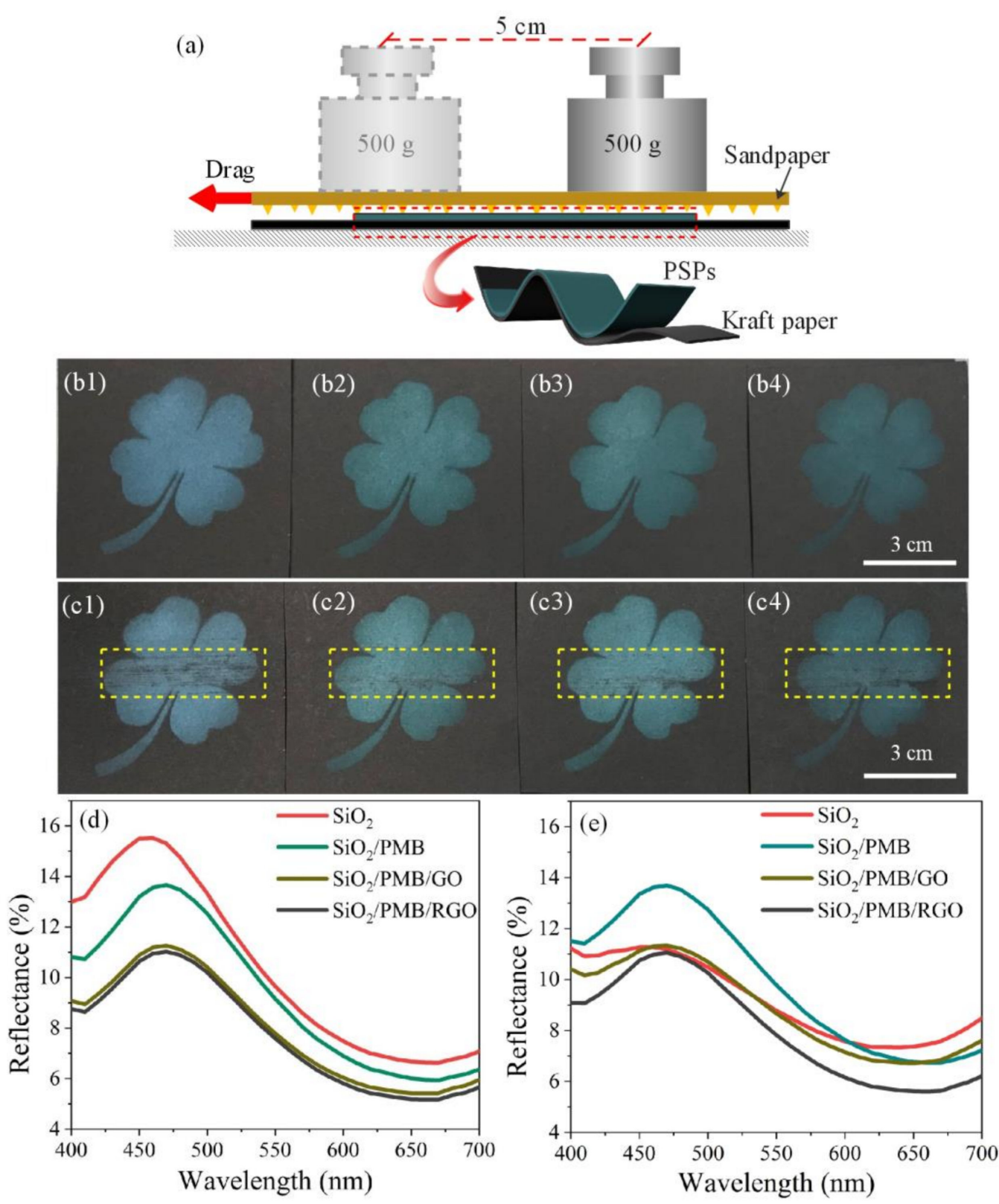

Figure 7. (a) Schematic illustration of abrasion test. (b,c) PSPs coated four-leaf clover patterns (b) before and (c) after abrasion, (b1,c1) $\mathrm{SiO}_{2},(\mathbf{b} 2, \mathbf{c} 2) \mathrm{SiO}_{2} / \mathrm{PMB},(\mathbf{b} 3, \mathbf{c} 3) \mathrm{SiO}_{2} / \mathrm{PMB} / \mathrm{GO}$ and $(\mathbf{b} 4, \mathbf{c} 4)$ $\mathrm{SiO}_{2} / \mathrm{PMB} / \mathrm{RGO}\left(\mathrm{SiO}_{2} \mathrm{NPs}\right.$ size: $\left.215.6 \mathrm{~nm}\right)$. The yellow boxes represent the abrasion areas. (d,e) Reflectance spectra of $(\mathbf{b})$ and (c), respectively. 


\section{Conclusions}

In summary, we have demonstrated that GO and RGO enhanced noniridescent structural colors with mechanical robustness were facilely established by spray coating method. $\mathrm{SiO}_{2}$ PSPs coated patterns with $\mathrm{SiO}_{2} \mathrm{NPs}$ of different particle sizes possess various noniridescent structural colors. Since pure $\mathrm{SiO}_{2}$ photonic films suffer from relatively poor structural robustness and unstable structural colors, mechanical robustness of $\mathrm{SiO}_{2} \mathrm{PCF}$ on a smooth glass substrate with and without PMB additive has been studied to clearly manifest the adhesive property of PMB. The mechanical stability of $\mathrm{SiO}_{2}, \mathrm{SiO}_{2} / \mathrm{PMB}$, $\mathrm{SiO}_{2} / \mathrm{PMB} / \mathrm{GO}, \mathrm{SiO}_{2} / \mathrm{PMB} / \mathrm{RGO}$ PSPs coated patterns has also been examined by the test of sandpaper abrasion, the four-leaf clover patterns with the addition of PMB exhibited better abrasion repellence. The inclusion of GO and RGO improved the color saturation of PSPs coated patterns and helped avoid whitish colors arisen from pure $\mathrm{SiO}_{2} \mathrm{PSP}$. This study provides a feasible approach for quickly constructing noniridescent structural colors and has potential practical applications in painting, optical displays.

Supplementary Materials: The following are available online at https://www.mdpi.com/article/10 .3390 / nano11040949/s1, Figure S1: TEM images of $\mathrm{SiO}_{2}$ nanoparticles with particle sizes of $294.0 \mathrm{~nm}$, scale bars are (a) $100 \mathrm{~nm}$, (b) $1 \mu \mathrm{m}$ and (c) $100 \mathrm{~nm}$, respectively. (d) The corresponding particle size distribution from TEM image measured by Nano Measurer software, Figure S2: DSC thermogram of PMB, Figure S3: FTIR spectrum of (a) PMB, (b) $\mathrm{SiO}_{2}$ and $\mathrm{SiO}_{2} / \mathrm{PMB}$ PSPs, (c) GO, RGO, (d) $\mathrm{SiO}_{2} / \mathrm{PMB} / \mathrm{GO}$ and $\mathrm{SiO}_{2} / \mathrm{PMB} / \mathrm{RGO}$ PSPs.

Author Contributions: Conceptualization, J.Y., C.-H.L. and C.-W.K.; methodology, J.Y.; software, J.Y.; validation, J.Y., C.-H.L. and C.-W.K.; formal analysis, J.Y.; investigation, J.Y. and C.-H.L.; data curation, J.Y.; funding acquisition, C.-W.K.; resources, C.-W.K.; supervision, C.-W.K.; visualization, J.Y.; writing-original draft, J.Y.; writing—review and editing, C.-H.L. and C.-W.K. All authors have read and agreed to the published version of the manuscript.

Funding: This work was funded by The Hong Kong Polytechnic University with grant number RHQG.

Data Availability Statement: Data is contained within the article.

Acknowledgments: The authors gratefully appreciate and acknowledge the financial support from The Hong Kong Polytechnic University.

Conflicts of Interest: The authors declare no conflict of interest.

\section{References}

1. Kim, J.B.; Lee, S.Y.; Lee, J.M.; Kim, S.H. Designing Structural-Color Patterns Composed of Colloidal Arrays. ACS Appl. Mater. Inter. 2019, 11, 14485-14509. [CrossRef]

2. Li, Q.; Zhang, Y.; Shi, L.; Qiu, H.; Zhang, S.; Qi, N.; Hu, J.; Yuan, W.; Zhang, X.; Zhang, K.Q. Additive Mixing and Conformal Coating of Noniridescent Structural Colors with Robust Mechanical Properties Fabricated by Atomization Deposition. ACS Nano 2018, 12, 3095-3102. [CrossRef] [PubMed]

3. Liu, P.; Chen, J.; Zhang, Z.; Xie, Z.; Du, X.; Gu, Z. Bio-inspired robust non-iridescent structural color with self-adhesive amorphous colloidal particle arrays. Nanoscale 2018, 10, 3673-3679. [CrossRef] [PubMed]

4. Yuan, W.; Zhou, N.; Shi, L.; Zhang, K.Q. Structural Coloration of Colloidal Fiber by Photonic Band Gap and Resonant Mie Scattering. ACS Appl. Mater. Inter. 2015, 7, 14064-14071. [CrossRef]

5. García, P.D.; Sapienza, R.; Blanco, Á.; López, C. Photonic Glass: A Novel Random Material for Light. Adv. Mater. 2007, 19, 2597-2602. [CrossRef]

6. Garcia, P.D.; Sapienza, R.; Lopez, C. Photonic glasses: A step beyond white paint. Adv. Mater. 2010, 22, 12-19. [CrossRef]

7. Zhao, Q.; Finlayson, C.E.; Schaefer, C.G.; Spahn, P.; Gallei, M.; Herrmann, L.; Petukhov, A.V.; Baumberg, J.J. Nanoassembly of Polydisperse Photonic Crystals Based on Binary and Ternary Polymer Opal Alloys. Adv. Opt. Mater. 2016, 4, 1494-1500. [CrossRef]

8. Yadav, A.; Gerislioglu, B.; Ahmadivand, A.; Kaushik, A.; Cheng, G.J.; Ouyang, Z.; Wang, Q.; Yadav, V.S.; Mishra, Y.K.; Wu, Y.; et al. Controlled self-assembly of plasmon-based photonic nanocrystals for high performance photonic technologies. Nano Today 2021, 37, 101072. [CrossRef]

9. Hou, J.; Li, M.; Song, Y. Patterned Colloidal Photonic Crystals. Angew. Chem. Int. Ed. 2018, 57, 2544-2553. [CrossRef] [PubMed]

10. Katagiri, K.; Tanaka, Y.; Uemura, K.; Inumaru, K.; Seki, T.; Takeoka, Y. Structural color coating films composed of an amorphous array of colloidal particles via electrophoretic deposition. NPG Asia Mater. 2017, 9, e355. [CrossRef] 
11. Liu, Z.; Zhang, Q.; Wang, H.; Li, Y. Structurally colored carbon fibers with controlled optical properties prepared by a fast and continuous electrophoretic deposition method. Nanoscale 2013, 5, 6917-6922. [CrossRef] [PubMed]

12. Xue, Y.; Wang, F.; Qin, Y.; Lu, B.; Wang, L.; Zhu, J. Angle-Independent Structurally Colored PS@TiO2 Film with Excellent Underwater Superoleophobicity in Harsh Environments. Langmuir 2019, 35, 6956-6961. [CrossRef] [PubMed]

13. Takeoka, Y.; Watanabe, M. Template Synthesis and Optical Properties of Chameleonic Poly (N-isopropylacrylamide) Gels Using Closest-Packed Self-Assembled Colloidal Silica Crystals. Adv. Mater. 2003, 15, 199-201. [CrossRef]

14. Ge, D.; Yang, L.; Wu, G.; Yang, S. Spray coating of superhydrophobic and angle-independent coloured films. Chem. Commun. 2014, 50, 2469-2472. [CrossRef]

15. Shen, Z.; Shi, L.; You, B.; Wu, L.; Zhao, D. Large-scale fabrication of three-dimensional ordered polymer films with strong structure colors and robust mechanical properties. J. Mater. Chem. 2012, 22, 8069. [CrossRef]

16. Harun-Ur-Rashid, M.; Bin Imran, A.; Seki, T.; Ishii, M.; Nakamura, H.; Takeoka, Y. Angle-independent structural color in colloidal amorphous arrays. Chem. Phys. Chem. 2010, 11, 579-583. [CrossRef]

17. Chung, K.; Yu, S.; Heo, C.J.; Shim, J.W.; Yang, S.M.; Han, M.G.; Lee, H.S.; Jin, Y.; Lee, S.Y.; Park, N.; et al. Flexible, angleindependent, structural color reflectors inspired by Morpho butterfly wings. Adv. Mater. 2012, 24, 2375-2379. [CrossRef]

18. Xu, F.; Zhong, L.; Xu, Y.; Feng, S.; Zhang, C.; Zhang, F.; Zhang, G. Highly efficient flame-retardant kraft paper. J. Mater. Sci. 2019, 54, 1884-1897. [CrossRef]

19. Kohri, M.; Nannichi, Y.; Taniguchi, T.; Kishikawa, K. Biomimetic non-iridescent structural color materials from polydopamine black particles that mimic melanin granules. J. Mater. Chem. C 2015, 3, 720-724. [CrossRef]

20. Lim, C.H.; Kang, H.; Kim, S.H. Colloidal assembly in Leidenfrost drops for noniridescent structural color pigments. Langmuir 2014, 30, 8350-8356. [CrossRef] [PubMed]

21. Takeoka, Y.; Yoshioka, S.; Takano, A.; Arai, S.; Nueangnoraj, K.; Nishihara, H.; Teshima, M.; Ohtsuka, Y.; Seki, T. Production of colored pigments with amorphous arrays of black and white colloidal particles. Angew. Chem. 2013, 125, 7402-7406. [CrossRef]

22. Kohri, M.; Yamazaki, S.; Kawamura, A.; Taniguchi, T.; Kishikawa, K. Bright structural color films independent of background prepared by the dip-coating of biomimetic melanin-like particles having polydopamine shell layers. Colloids Surf. A Physicochem. Eng. Asp. 2017, 532, 564-569. [CrossRef]

23. Geim, A.K.; Novoselov, K.S. The rise of graphene. Nat. Mater. 2007, 6, 183-191. [CrossRef] [PubMed]

24. Kim, H.; Abdala, A.A.; Macosko, C.W. Graphene/polymer nanocomposites. Macromolecules 2010, 43, 6515-6530. [CrossRef]

25. Chung, C.; Kim, Y.K.; Shin, D.; Ryoo, S.R.; Hong, B.H.; Min, D.H. Biomedical applications of graphene and graphene oxide. Acc. Chem. Res. 2013, 46, 2211-2224. [CrossRef]

26. Morales-Narváez, E.; Merkoçi, A. Graphene oxide as an optical biosensing platform. Adv. Mater. 2012, 24, 3298-3308. [CrossRef]

27. Ahmadivand, A.; Gerislioglu, B.; Ramezani, Z. Gated graphene island-enabled tunable charge transfer plasmon terahertz metamodulator. Nanoscale 2019, 11, 8091-8095. [CrossRef]

28. Pursiainen, O.L.; Baumberg, J.J.; Winkler, H.; Viel, B.; Spahn, P.; Ruhl, T. Nanoparticle-tuned structural color from polymer opals. Opt. Express 2007, 15, 9553-9561. [CrossRef]

29. Kredel, J.; Gallei, M. Compression-Responsive Photonic Crystals Based on Fluorine-Containing Polymers. Polymers 2019, 11, 2114. [CrossRef]

30. Schäfer, C.G.; Gallei, M.; Zah, J.T.; Engelhardt, J.; Hellmann, G.P.; Rehahn, M. Reversible light-, thermo-, and mechano-responsive elastomeric polymer opal films. Chem. Mater. 2013, 25, 2309-2318. [CrossRef]

31. Li, Y.; Zhou, L.; Liu, G.; Chai, L.; Fan, Q.; Shao, J. Study on the fabrication of composite photonic crystals with high structural stability by co-sedimentation self-assembly on fabric substrates. Appl. Surf. Sci. 2018, 444, 145-153. [CrossRef]

32. Yuan, W.; Li, Q.; Zhou, N.; Zhang, S.; Ding, C.; Shi, L.; Zhang, K.Q. Structural Color Fibers Directly Drawn from Colloidal Suspensions with Controllable Optical Properties. ACS Appl. Mater. Interfaces 2019, 11, 19388-19396. [CrossRef]

33. Liu, P.; Bai, L.; Yang, J.; Gu, H.; Zhong, Q.; Xie, Z.; Gu, Z. Self-assembled colloidal arrays for structural color. Nanoscale Adv. 2019, 1, 1672-1685. [CrossRef]

34. Wu, X.; Hong, R.; Meng, J.; Cheng, R.; Zhu, Z.; Wu, G.; Li, Q.; Wang, C.F.; Chen, S. Hydrophobic Poly(tert-butyl acrylate) Photonic Crystals towards Robust Energy-Saving Performance. Angew. Chem. Int. Ed. Engl. 2019, 58, 13556-13564. [CrossRef]

35. Liao, J.; Zhu, C.; Gao, B.; Zhao, Z.; Liu, X.; Tian, L.; Zeng, Y.; Zhou, X.; Xie, Z.; Gu, Z. Multiresponsive Elastic Colloidal Crystals for Reversible Structural Color Patterns. Adv. Funct. Mater. 2019, 29, 1902954. [CrossRef]

36. Zhou, C.; Qi, Y.; Zhang, S.; Niu, W.; Ma, W.; Wu, S.; Tang, B. Rapid fabrication of vivid noniridescent structural colors on fabrics with robust structural stability by screen printing. Dye. Pigm. 2020, 176, 108226. [CrossRef]

37. Kuznetsov, A.I.; Miroshnichenko, A.E.; Brongersma, M.L.; Kivshar, Y.S.; Luk'yanchuk, B. Optically resonant dielectric nanostructures. Science 2016, 354, 2472. [CrossRef]

38. Fu, Y.H.; Kuznetsov, A.I.; Miroshnichenko, A.E.; Yu, Y.F.; Luk'yanchuk, B. Directional visible light scattering by silicon nanoparticles. Nat. Commun. 2013, 4, 1527. [CrossRef] [PubMed]

39. Urban, M.W.; Davydovich, D.; Yang, Y.; Demir, T.; Zhang, Y.; Casabianca, L. Key-and-lock commodity self-healing copolymers. Science 2018, 362, 220-225. [CrossRef]

40. Strankowski, M.; Włodarczyk, D.; Piszczyk, Ł.; Strankowska, J. Polyurethane nanocomposites containing reduced graphene oxide, FTIR, Raman, and XRD studies. J. Spectrosc. 2016, 2016, 7520741. [CrossRef] 
41. Emiru, T.F.; Ayele, D.W. Controlled synthesis, characterization and reduction of graphene oxide: A convenient method for large scale production. Egypt. J. Basic Appl. Sci. 2017, 4, 74-79. [CrossRef]

42. Sharma, N.; Sharma, V.; Jain, Y.; Kumari, M.; Gupta, R.; Sharma, S.K.; Sachdev, K. Synthesis and characterization of graphene oxide (GO) and reduced graphene oxide (rGO) for gas sensing application. Macromol. Symp. 2017, 376, 1700006. [CrossRef]

43. Zhang, T.Y.; Zhang, D. Aqueous colloids of graphene oxide nanosheets by exfoliation of graphite oxide without ultrasonication. Bull. Mater. Sci. 2011, 34, 25-28. [CrossRef] 